\title{
Dedifferentiated liposarcoma of the thyroid gland: A case report
}

\author{
YO KISHIMOTO $^{1}$, AYAMI OHNO KISHIMOTO ${ }^{2}$, YOSUKE YAMADA ${ }^{3}$, MASAYUKI KITANO $^{4}$, YUJI KITADA ${ }^{5}$, \\ MORIMASA KITAMURA $^{1}$, ICHIRO TATEYA ${ }^{1}$, MAKOTO SONOBE ${ }^{6}$ and KOICHI OMORI ${ }^{1}$ \\ Departments of ${ }^{1}$ Otolaryngology-Head and Neck Surgery, and ${ }^{2}$ Diagnosic Imaging and Nuclear Medicine; \\ ${ }^{3}$ Department of Diagnostic Pathology, Graduate School of Medicine, Kyoto University, Kyoto 606-8507; \\ ${ }^{4}$ Department of Otolaryngology-Head and Neck Surgery, Japanese Red Cross Osaka Hospital, Osaka 543-8555; \\ ${ }^{5}$ Department of Otolaryngology-Head and Neck Surgery, Kitano Hospital Tazuke Medical Research Institute, \\ Osaka 530-8480; ${ }^{6}$ Department of Thoracic Surgery, Graduate School of Medicine, \\ Kyoto University, Kyoto 606-8507, Japan
}

Received September 28, 2018; Accepted April 5, 2019

DOI: $10.3892 / \mathrm{mco} .2019 .1882$

\begin{abstract}
Liposarcoma is one of the most common soft tissue tumors that affect adults. Liposarcoma is typically identified in the retroperitoneum, trunk and extremities as a slow-growing mass, yet is rare in the thyroid gland. Herein, we report a case of dedifferentiated liposarcoma that developed in the thyroid gland, and performed a literature review. A 66-year-old male with a history of well-differentiated liposarcoma in the thymus presented with a rapidly enlarging mass in the thyroid gland. Malignancy was suspected due to the local extension of the tumor, and complete resection of the tumor was achieved. Tissue diagnosis from the tumor following surgery revealed that dedifferentiated liposarcoma, and tumor progression was difficult to control with adjuvant radiation and chemotherapy. This is, to the best of our knowledge, the first case to report dedifferentiated liposarcoma in the thyroid gland, and the difficulty in initial diagnosis as well as the aggressive features of dedifferentiated liposarcoma are highlighted.
\end{abstract}

\section{Introduction}

Liposarcoma (LPS) is one of the most common soft tissue neoplasms of adults, and accounts for approximately $20 \%$ of all sarcomas $(1,2)$. This tumor of mesenchymal origin is usually found in the retroperitoneum, trunk, and extremities

Correspondence to: Dr Yo Kishimoto, Department of Otolaryngology-Head and Neck Surgery, Graduate School of Medicine, Kyoto University, 54 Kawahara-cho, Kyoto 606-8507, Japan

E-mail: y_kishimoto@ent.kuhp.kyoto-u.ac.jp

Key words: liposarcoma, dedifferentiated liposarcoma, thyroid gland, malignant tumor, head and neck tumor as a slow-growing and painless mass developing during the middle decades of life (1). Furthermore, there are often multiple satellite lesions extending beyond the boundary of the primary tumor (3).

In the head and neck region, LPS is rare and accounts for only $2 \%$ of all cases (4). Rarer still is the presence of LPS in the thyroid, and only 11 cases of primary LPS (2,5-11) and 4 cases of metastatic LPS in the thyroid gland have been reported (3,12-14).

Herein we report the first case of dedifferentiated LPS (DDLPS) in the thyroid gland of a 66-year-old male, and discuss its clinical course; additionally, we performed a literature review.

\section{Case report}

Written informed consent to report the pathology of this case was obtained from the patient. A 66-year-old male was referred to our hospital with a one-month history of dysphagia. He also noticed an enlarging anterior neck mass a few days before presentation. The patient had undergone resection of a well-differentiated LPS (WDLPS) arising in the thymus 5 years previously.

At the time of consultation, physical examination demonstrated a $40 \mathrm{~mm}$ firm, rubbery, and fixed mass in the right lobe of the thyroid gland, and screening laboratory tests demonstrated an euthyroid state and a normal serum thyroglobulin level. Ultrasound was performed and revealed a 42×30x27 mm heterogeneous nodule with poorly defined margins occupying the right lobe of the thyroid gland. Ultrasound-guided fine needle aspiration cytology identified spindle cells. While nuclear enlargement and a clear nuclear body were observed, cytological atypia was not prominent, and there was low suspicion for WDLPS.

The vocal folds were initially unaffected; however, the patient started to complain of hoarseness a few weeks after the first visit, and a right recurrent laryngeal nerve paralysis was observed. While the membranous portion of the trachea 
was compressed anteriorly by the mass, there was no apparent tumor invasion into the trachea or esophagus by endoscopic examination.

Contrast-enhanced computed tomography (CT) imaging revealed the $40 \mathrm{~mm}$ mass with heterogeneous enhancement occupying the entire right lobe of the thyroid gland. The margin of the tumor was not clear and the tumor appeared to invade the trachea and the esophagus (Fig. 1). Enlarged right internal jugular lymph nodes were also detected. Magnetic resonance imaging (MRI) revealed an enhancing mass with irregular margins in the right lobe of the thyroid gland. Centrally, the tumor demonstrated low signal intensity on T2 weighted images (T2WI) and showed almost no enhancement on contrast-enhanced $\mathrm{T} 1$ weighted images (T1WI). The tumor appeared fibrotic, and no lipomatous portion was detected (Fig. 2). Fluorodeoxyglucose-positron emission tomography (FDG-PET) showed a hypermetabolic mass in the right lobe of the thyroid gland. No evidence of distant metastases or recurrent thymus tumor was detected (Fig. 3).

Although FNA showed no evidence of cellular atypia, malignancy was suspected because of the local extension of the tumor, and total thyroidectomy with right modified radical neck dissection as well as tracheal and esophageal resections were performed.

A $7 \mathrm{~cm}$ tumor was identified in the right lobe infiltrating the second tracheal ring, the esophageal muscle, and the right recurrent laryngeal nerve. Furthermore, the tumor was surrounded by fibrous tissue extending from the mediastinum into the larynx/pharynx and the common carotid artery, and frozen section of the fibrous tissue revealed spindle cells. The tumor was resected with the right side of the 1st to 3rd tracheal ring, the esophageal and cricopharyngeus muscles, and the right recurrent laryngeal nerve and upper mediastinal dissection was performed. Even though the extensive local invasion of the tumor over the neck and mediastinum, grossly complete resection of the tumor was achieved.

Histological examination showed hypercellularity and infiltrative overgrowth of spindle cells with nuclear atypia. The right lobe was completely replaced by the tumor without any lipid-laden cells, and the central part of the tumor was replaced by fibrotic tissue. The tumor lacks epithelial component and cells were rarely stained with $\mathrm{p} 53$. These findings didn't support a diagnosis of anaplastic carcinoma of the thyroid. Further, the tumor didn't express S100, desmin, $\alpha$-smooth muscle actin, myogenin, myoglobin, and CD34 suggesting a dedifferentiated tumor. Overexpression of human murine double minute 2 (MDM2) and cyclin D kinase 4 (CKD4) were detected, and the tumor was diagnosed as DDLPS with cervical lymph node metastases (Fig. 4).

Postoperatively, the patient received adjuvant radiation ( 22 Gy in 11 fractions +35 Gy in 14 fractions delivered to the operative field) and chemotherapy (4 courses of $1,200 \mathrm{mg} / \mathrm{m}^{2}$ gemcitabine and $90 \mathrm{mg} / \mathrm{m}^{2}$ docetaxel). Two months postoperatively, however, the patient presented with growth of the residual disease in the neck. Four months postoperatively, metastases to the psoas muscle, as well as to the axillary and intra-thoracic lymph nodes were identified (Fig. 5). Nine months postoperatively, the patient died from severe bleeding due to local tumor progression in the neck.
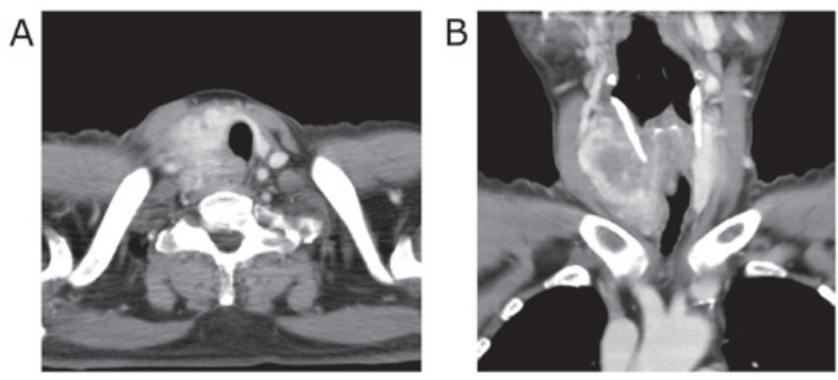

Figure 1. Preoperative CT findings. The right lobe of the thyroid gland was occupied by the tumor and demonstrated heterogeneous enhancement. (A: axial, B: coronal).
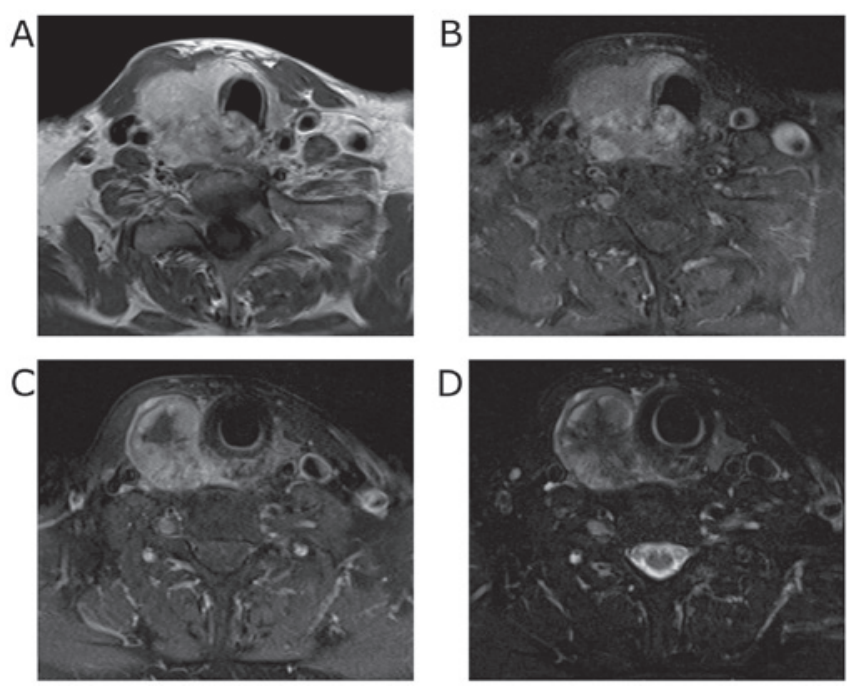

Figure 2. MRI findings. A well-enhanced tumor with unclear margins was observed in the right lobe of the thyroid gland. (A) contrast enhanced-T1WI, (B) T1-SPAIR image, (C) T1-SPAIR image, (D) T2-SPAIR image.
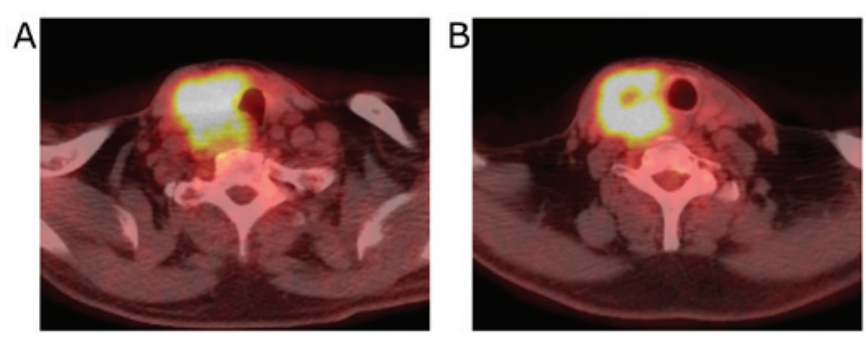

Figure 3. PET findings. (A) A hyper-metabolic tumor was observed in the right lobe of the thyroid gland. (B) The central part of the tumor exhibited no uptake of FDG.

\section{Discussion}

In the head and neck region, LPS is very rare and represents only $1 \%$ of head and neck sarcomas (15). Correct diagnosis is difficult before surgical resection, and Davis reported that one third of patients with head and neck LPS had an initial pathologic misdiagnosis (4). Incorrect diagnosis may lead to delayed or inadequate treatment. In our case, the patient was initially suspected to have anaplastic thyroid carcinoma or lymphoma because of the rapid extension of the tumor. Recurrence of WDLPS of the thymus was not considered 

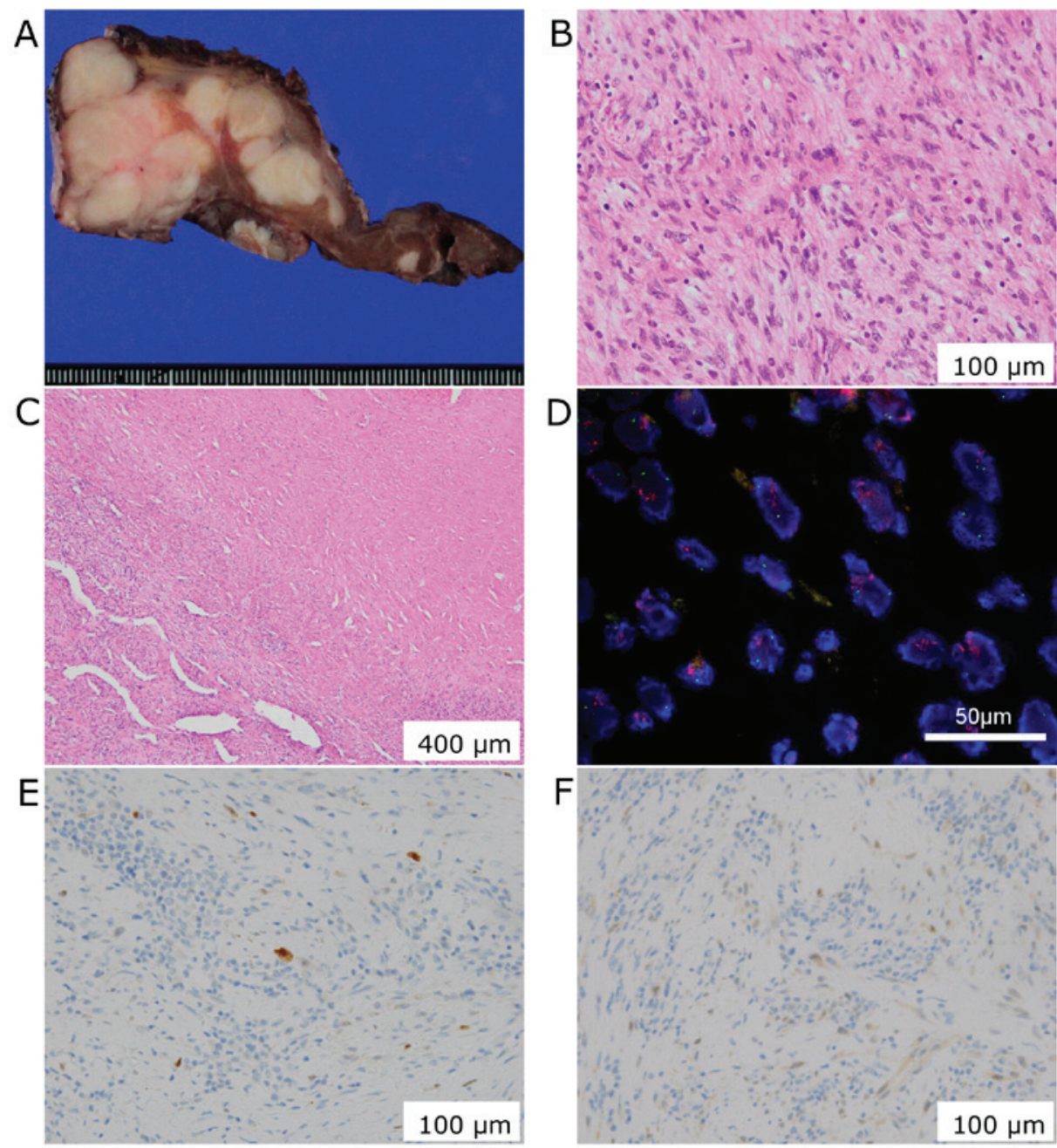

Figure 4. Histological findings. In the resected specimen, (A) the tumor appears multi-nodular, and (B) photomicrograph (hematoxylin-eosin staining) reveals dedifferentiated spindle cells (B) with fibrosis (C) $400 \mu \mathrm{m}$. Fluorescence in situ hybridization (FISH) shows MDM2 gene amplification (D, red) $50 \mu \mathrm{m}$, and immunohistochemistry reveals the expression of MDM2 (E) and CDK4 (F) $100 \mu \mathrm{m}$.
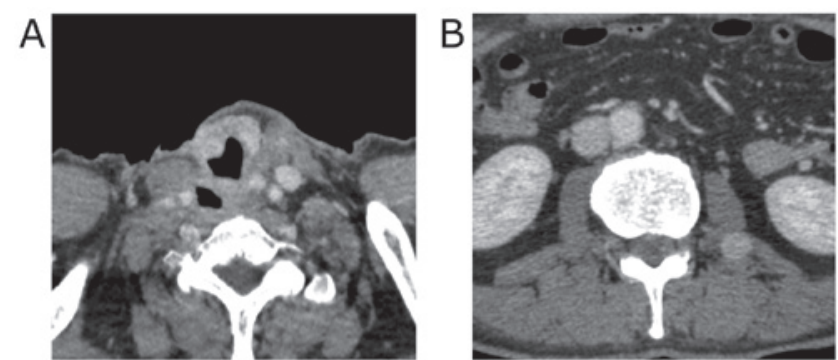

Figure 5. Postoperative CT findings. Growth of the residual disease in the trachea (A) and metastases to the psoas muscle (B) were identified.

because of the long interval from the initial resection of that tumor. However, according to previous reports summarized in the Table I, LPS can recur long after the initial resection. In a patient with a history of LPS, the possibility of a recurrence or metastasis should be considered, even if the suspect lesion is in the thyroid.

LPSs are mostly idiopathic and etiology of LPS still remains unclear. Similar to other sarcomas, complex karyotypic defects leading to genetic instability and disturbances in cell cycle genes are reported to cause LPSs (16). The complex karyotypic defects can be induced by radiation, however, LPSs are less frequent in radiation induced sarcomas. Further, LPSs are less frequently seen in patients with genetic syndromes than in patients with sporadic soft tissue sarcomas (17).

According to the classification of the World Health Organization, LPSs are a heterogeneous group classified into four subtypes based on morphology and genetic findings, namely: Atypical lipomatous tumors (ALT)/WDLPS, DDLPS, myxoid LPS (MLPS), and pleomorphic LPS (PLS) (18). Each subtype exhibits different clinical behaviors. DDLPS, first described by Evans in 1979 (19), is characterized by the transition from an adipocyte-rich, well-differentiated region within a tumor to a non-lipogenic, spindle cell-rich region. It develops de novo in most cases, and $25-40 \%$ of patients show progression from ALT/WDLPS to DDLPS. Although ALT/WDLPS has a low metastatic potential, DDLPS shows a strong propensity for distant lung metastasis and local recurrence $(18,20)$. In the present case, the tumor extended rapidly and adjuvant radiation and chemotherapy were not effective in halting its progression, consistent with features of DDLPS. It is difficult to determine whether the tumor was a local recurrence or metastasis of the original LPS of the thymus, particularly because there appeared to be infiltration 


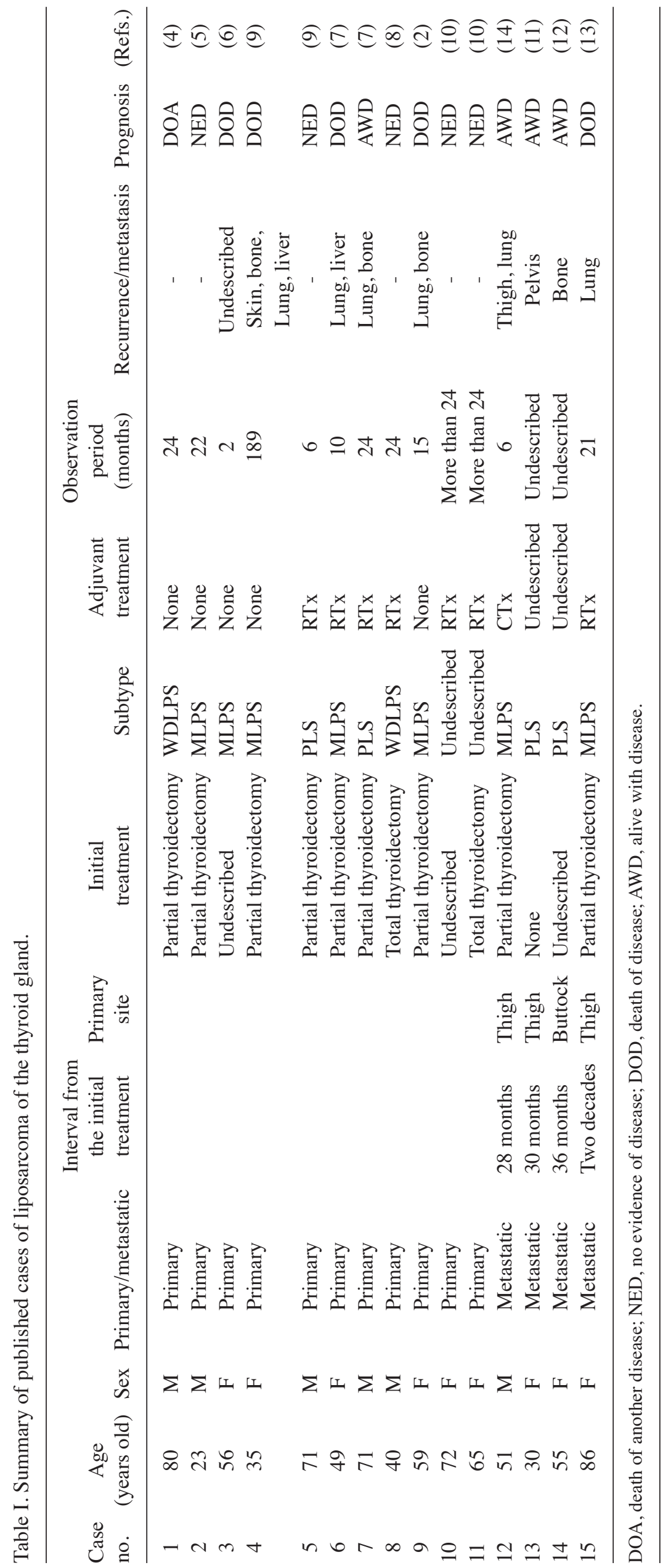


of the tumor into the mediastinum. We thought of this case as metastasis as main lesion was located in the thyroid gland and not in mediastinum.

DDLPS shares radiologic features with WDLPS, and dedifferentiation is usually suggested by the presence of a focal, nodular, nonlipomatous region greater than $10 \mathrm{~mm}$ in size (21). These non-adipose foci are easily detected on MRI. Although WDLPS shows high signal intensities on both T1WI and T2WI, dedifferentiated regions appear to show low intensity area on both sequences (22). In our case, because of its aggressive characteristics, anaplastic carcinoma or lymphoma was initially suspected. Usually, anaplastic carcinoma of the thyroid gland is heterogeneous with areas of necrosis and mixed signal on T1 and T2WI and moderate-to-marked enhancement, and lymphoma has homogenous mild enhancement and mild T2 hyperintense signal compared with the surrounding normal thyroid tissues (23). As anaplastic carcinoma and DDLPS share some features on MRI, it is difficult to distinguish between them preoperatively.

Histologically, ALT/WDLPS and DDLPS are characterized by the amplification of the 12q13-15 chromosome region encoding for potential oncogenes including mouse double minute 2 (MDM2) and cyclin dependent kinase-4 (CDK4). DDLPS is characterized by the transition of regions of the tumor from adipocyte-rich, well-differentiated cells to non-lipogenic, spindle cell-rich cells (18). In this case, any lipid-laden cells were not observed in the tumor and it was partly replaced by fibrotic tissues as expected from the pre-operative images.

Standard treatment for DDLPS is wide surgical excision, which is frequently followed by radiation. Adjuvant chemotherapy is often performed as well. Even with multidisciplinary treatment, however, DDLPS often recurs locally and rapidly, and can metastasize to lung, bone, or liver, and disease progression is difficult to control (21). Recently, molecular therapies including tyrosine kinase inhibitors, mouse double minute 2 (MDM2) antagonists, cyclin dependent kinase-4 (CDK4) antagonists, peroxisome proliferator-activated receptor gamma (PPAR- $\gamma$ ) agonists, and Nelfinavir have been shown to have some therapeutic effects on DDLPS (18), and further clinical studies are warranted to establish novel therapeutic strategies for DDLPS.

We have presented, to our knowledge, the first case of dedifferentiated LPS of the thyroid gland. The case presented difficulties in regards to initial diagnosis, and demonstrated the very aggressive features of DDLPS despite aggressive surgery and chemoradiation. To ensure timely and accurate initial diagnosis, the differential of any thyroid mass must be a possible metastatic lesion, particularly in a patient with a history of previous malignancy. Novel therapeutic strategies are needed for better outcomes in patients with DDLPS.

\section{Acknowledgements}

Not applicable.

\section{Funding}

No funding was received.

\section{Availability of data and materials}

All data generated or analyzed during the present study are included in this published article.

\section{Authors' contributions}

YKis acquired the data, performed the literature review and wrote the manuscript. MK, YKit, MK, IT, MS, KO acquired the data and contributed clinical advice. AKO evaluated the images and YY evaluated the specimens. All authors read and approved the final manuscript.

\section{Ethics and consent to participate}

Not applicable.

\section{Patient consent for publication}

Written informed consent was obtained for the publication of data and materials.

\section{Competing interests}

The authors declare that they have no competing interests.

\section{References}

1. Blumberg JM, Jedrych J, Costa J and Judson B: Cervical dedifferentiated liposarcoma with meningothelial-like whorling. Head Neck Pathol 6: 476-480, 2012.

2. Huang GW, Li YX and Hu ZL: Primary myxoid liposarcoma of the thyroid gland. J Clin Pathol 62: 1037-1038, 2009.

3. Azar AR, Weynand B, Daumerie C and Coche E: Metastatic liposarcoma of the thyroid gland. Br J Radiol 76: 750-752, 2003.

4. Davis EC, Ballo MT, Luna MA, Patel SR, Roberts DB, Nong X and Sturgis EM: Liposarcoma of the head and neck: The University of Texas M. D. Anderson Cancer Center experience. Head Neck 31: 28-36, 2009.

5. Nielsen VT, Knudsen N and Holm IE: Liposarcoma of the thyroid gland. Tumori 72: 499-502, 1986.

6. Griem KL, Robb PK, Caldarelli DD and Templeton AC: Radiation-induced sarcoma of the thyroid. Arch Otolaryngol Head Neck Surg 115: 991-993, 1989.

7. Andrion A, Gaglio A, Dogliani N, Bosco E and Mazzucco G: Liposarcoma of the thyroid gland. Fine-needle aspiration cytology, immunohistology, and ultrastructure. Am J Clin Pathol 95: 675-679, 1991.

8. Mitra A, Fisher C, Rhys-Evans P and Harmer C: Liposarcoma of the thyroid. Sarcoma 8: 91-96, 2004.

9. Kilic M, Keskek M, Albayrak L, Ertan T, Gocmen E and Koc M: Liposarcoma of the thyroid gland: A case report. Acta Chir Belg 107: 73-74, 2007.

10. Awad WI, Evans PHR, Nicholson AG and Goldstraw P: Liposarcoma of the thyroid gland mimicking retrosternal goiter. Ann Thorac Surg 75: 566-568, 2003.

11. Kumar V, Raj A and Rathore PK: Liposarcoma of thyroid gland: A review of the gained experience. Indian J Cancer 51: 548-549, 2014.

12. Bashir H, Nawaz MK, Shah MA and Ahmad E: Pleomorphic liposarcoma metastatic to the thyroid gland. Clin Nucl Med 27: 9-10, 2002.

13. Brandwein-Gensler M, Urken $M$ and Wang B: Collision tumor of the thyroid: A case report of metastatic liposarcoma plus papillary thyroid carcinoma. Head Neck 26: 637-641, 2004.

14. Tysome JR, Sandison A and Clarke PM: Myxoid liposarcoma metastatic to the thyroid gland: A case report and literature review. J Laryngol Otol 120: 511-513, 2006.

15. Golledge J, Fisher C and Rhys-Evans PH: Head and neck liposarcoma. Cancer 76: 1051-1058, 1995.

16. Hui JY: Epidemiology and etiology of sarcomas. Surg Clin North Am 96: 901-914, 2016. 
17. Penel N, Grosjean J, Robin YM, Vanseymortier L, Clisant S and Adenis A: Frequency of certain established risk factors in soft tissue sarcomas in adults: A prospective descriptive study of 658 cases. Sarcoma 2008: 459386, 2008.

18. De Vita A, Mercatali L, Recine F, Pieri F, Riva N, Bongiovanni A, Liverani C, Spadazzi C, Miserocchi G, Amadori D, et al: Current classification, treatment options, and new perspectives in the management of adipocytic sarcomas. Onco Targets Ther 9: 6233-6246, 2016.

19. Evans HL: Liposarcoma: A study of 55 cases with a reassessment of its classification. Am J Surg Patho 3: 507-523, 1970.

20. Singer S, Antonescu CR, Riedel E and Brennan MF: Histologic subtype and margin of resection predict pattern of recurrence and survival for retroperitoneal liposarcoma. Ann Surg 238 : 358-370, 2003.
21. Murphey MD, Arcara LK and Fanburg-Smith J: Imaging of Musculoskeletal Liposarcoma with radiologic-pathologic correlation. RadioGraphics 25: 1371-1395, 2005.

22. Kito M, Yoshimura Y, Isobe K, Aoki K, Suzuki S, Tanaka A, Okamoto M, Sano K and Kato H: Clinical outcome of dedifferentiated liposarcoma in the extremities: A retrospective case series of 7 patients. J Orthop Sci 21: 673-677, 2016.

23. Aiken AH: Imaging of thyroid cancer. Semin Ultrasound CT MR 33: 138-149, 2012.

(i) (2) This work is licensed under a Creative Commons Attribution-NonCommercial-NoDerivatives 4.0 International (CC BY-NC-ND 4.0) License. 